\title{
Spatial analysis of the air pollutant index in the southern region of Peninsular Malaysia using environmetric techniques
}

\begin{abstract}
Air pollution is becoming a major environmental issue in the southern region of Peninsular Malaysia. Environmetric techniques (HACA, DA, and PCA/FA) were used to evaluate the spatial variations in the southern region of Peninsular Malaysia, followed by API prediction comparison using ANN and MLR models. The datasets of air pollutant parameters for 3 years (2005i 2007) were applied in this study. HACA clustered three different groups of similarity based on the characteristics of air quality parameters. DA shows all seven parameters $(\mathrm{CO}$, O3, PM10, SO2, NOx, NO, and NO2) gave the most significant variables after stepwise backward mode. PCA/FA identify that the major source of air pollution is due to combustion of fossil fuels in motor vehicles and industrial activities. The ANN model shows a better prediction compared to the MLR model with R2 values equal to 0.819 and 0.773 respectively. This study concluded that the environmetric techniques and modelling become an excellent tool in API assessment, air pollution source identification, apportionment, and interpretation of complex dataset with a view to get better information about the air quality, and can be setbacks in designing an API monitoring network for effective air pollution resources management.
\end{abstract}

Keyword: Air pollutant index; HACA; DA; PCA/FA; ANN; MLR 